Urologe $2022 \cdot 61: 133-141$

https://doi.org/10.1007/s00120-021-01734-y

Angenommen: 23. November 2021

Online publiziert: 22. Dezember 2021

(c) The Author(s), under exclusive licence to Springer Medizin Verlag $\mathrm{GmbH}$, ein Teil von Springer Nature 2021

\section{Virtual und Augmented Reality in der Urologie}

P. Sparwasser · M. Haack · L. Frey · A. Haferkamp · H. Borgmann

Department of Urology, University Medical Center, Johannes Gutenberg University, Mainz, Deutschland

\section{Zusammenfassung}

Zwar haben jeher technologische Weiterentwicklungen die medizinische Versorgung in deren stetigem Wandel optimiert, so waren diese jedoch immer noch für den Anwender weitestgehend fassbar. Getrieben durch immense finanzielle Anstrengungen sind innovative Produkte und technische Lösungen entstanden, die den medizinischen Alltag transformieren und diesen in Zukunft um eine Dimension erweitern werden: die Virtual und Augmented Reality. Dieser Übersichtsartikel fasst die aktuellen wissenschaftlichen Projekte und den zukünftigen Nutzen von Virtual und Augmented Reality im Fachgebiet der Urologie zusammen.

\section{Schlüsselwörter}

Mixed Reality · Datenbrille · Augmented Reality · Telemedizin · Matching
Prinzipiell muss zu Beginn unseres Übersichtsartikels festgehalten werden, dass sämtliche erforschten Anwendungen gerade im urologischen Fachbereich zwar meist einen subjektiven Nutzen oder Vorteil ausweisen, ohne dass jedoch zum gegenwärtigen Zeitpunkt belastbare und robuste Studien vorliegen, die dies belegen [1]. Der Trend jedoch, Virtual Reality (VR) und Augmented Reality (AR) in die klinische Routine integrieren zu wollen, ist zweifelsohne vorhanden und wohl nur eine Frage der Zeit. Während noch im Jahr 2000 auf der Metadatenbank PubMed nur 121 Artikel zu VR und 7 Artikel zu AR publiziert wurden, sind es nun jährlich mehr als > 2000 (VR) bzw. > 600 (AR) mit stetig steigender Tendenz (• Abb. 1).

Unter VR versteht man dabei die künstliche von Computern simulierte und generierte Umgebung, mit welcher eine Interaktion möglich ist [2]. Die VR ermöglicht es dadurch an einem Ort zu sein und gleichzeitig eine andere Umgebung, eine eben rein virtuelle Welt, wahrzunehmen.

Die AR hingegen generiert keine rein virtuelle Umgebung. Entsprechend des Begriffs „augmented“ (engl. „to augment something" = etwas erweitern) werden hier vielmehr künstliche Informationen, meist entsprechend visuellen oder auditiven Sensationen in die reale Welt integriert [2]. Die reale Welt bleibt somit weiterhin optimiert durch virtuelle Informationen fassbar.

\section{I) VR und AR werden zunehmend in die klinische Routine integriert}

Ein klassisches Beispiel hierfür ist die Projektion in das Sichtfeld von Bildern oder von virtuellen Modellen mittels einer Smartglass ( $\mathbf{A b b}$. 2). Eine Smartglass ist dabei eine Datenbrille mit integrierten Prozessoren, die Informationen zum Sichtfeld des Benutzers hinzufügen kann. Im Falle der AR behält der Anwender dabei freie Sicht auf die reale Welt und kann parallel auf digitale Informationen zurückgreifen. Je nach Smartglass-Modell kann diese allerdings auch eine rein virtuelle Umgebung, also eine VR kreieren. Borgmann et al. [3] definierten die potenziellen Anwendungsgebiete von Smartglasses im urologischen Operationssaal folgendermaßen:

- Foto-, Video- und Liveübertragung für Aus-, Fort- und Weiterbildung

- Fotos und Videos zur Dokumentation

- Telekonsultation von Kollegen 


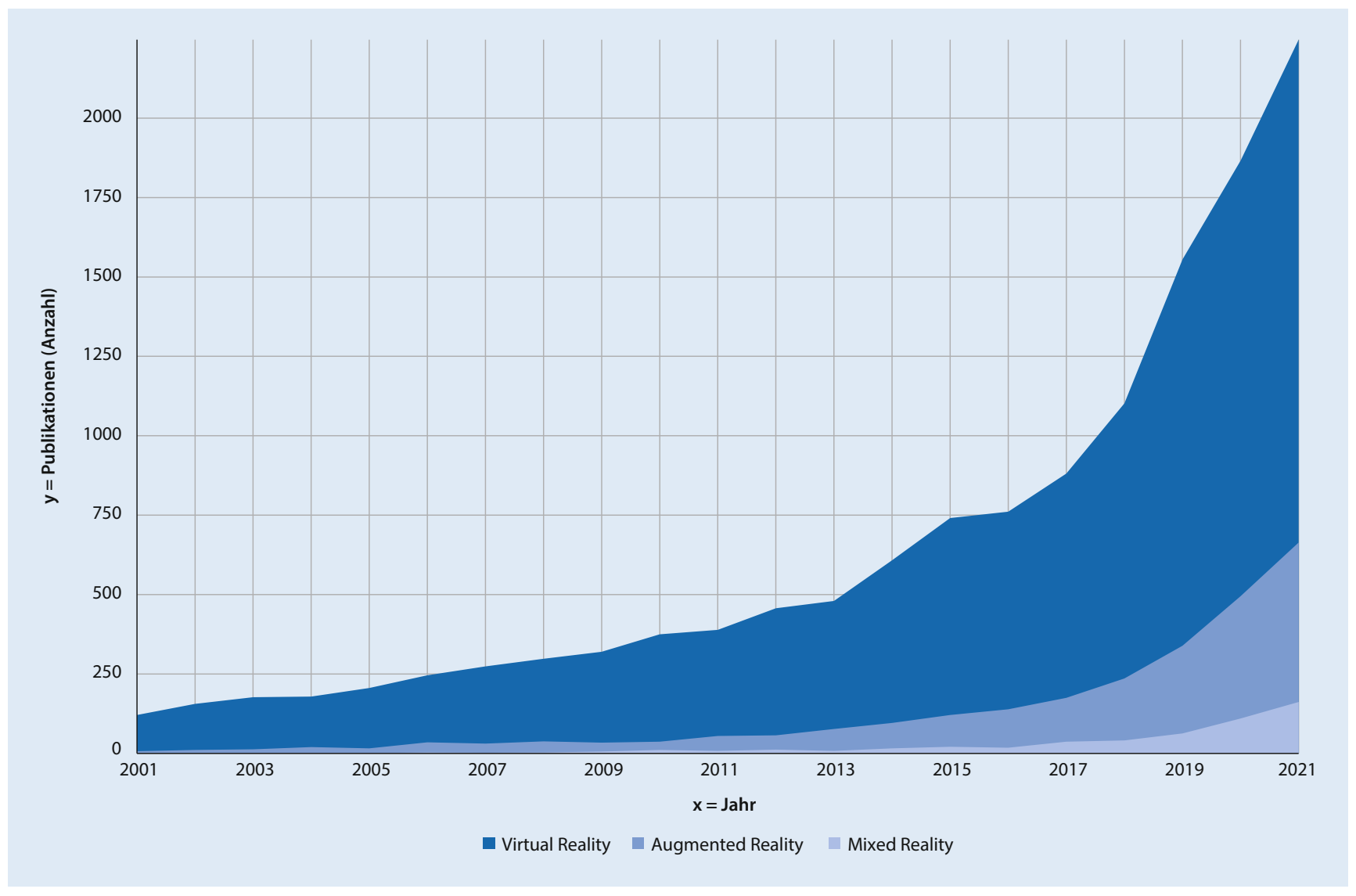

Abb. 1 A Anzahl wissenschaftlicher Publikationen auf der Metadatenbank PubMed mit Bezugnahme auf Virtual Reality (VR) und Augmented Reality (AR) in den Jahren 2000 bis 2021

- Aufrufen elektronischer Patientenakte

- Aufrufen von radiologischer Bildgebung.

Die Mixed Reality (MR) vereint meist mittels einer Smartglass Attribute dieser beiden Welten und ist entsprechend ein Hybrid aus realen und virtuellen Sensationen mit dynamischen Übergängen von VR in AR [4].

Während diese Begrifflichkeiten von VR, $A R$ und MR noch halbwegs verständlich sind ist es die sog. Metaverse nur noch bedingt. Zwar existiert dieser Begriff der Metaverse schon seit 1992 (Science-Fiction Roman Snow Crash von Neal Stephenson) jedoch hat er erst kürzlich im Rahmen des "rebrandings" von Facebook ${ }^{\circledR}$ zu Meta ${ }^{\circledR}$ mit dem Ziel eine neue technologische Epoche einzuleiten neue Aufmerksamkeit erlangt. Der CEO von Facebook Mark Zuckerberg selbst beschreibt dabei Metaverse wenig verständlich folgendermaßen: „Man kann sich das Metaverse als verkörpertes Internet vorstellen, in dem man Inhalte nicht nur anschaut, sondern in ihnen steckt" [5]. Im Metaverse verschwimmen und verschmelzen präziser formuliert VR und AR mit dem Internet zu einem kollektiven interaktiven dreidimensionalen (3D-)Universum, wobei der Nutzer diesen interaktiven Raum als real empfindet, indem er dort als Avatar "tatsächlich" alltägliche Dinge wie Arbeiten, Einkaufen, Reisen, Konzertbesuche etc. erleben kann.

\section{I) Im Metaverse verschmelzen \\ VR und AR mit dem Internet zu einem kollektiven interaktiven dreidimensionalen Universum}

Stand heute ist davon auszugehen, dass viele Anwendungen im Bereich der VR, AR und MR ihren Nutzen im medizinischen Alltag beweisen werden, während man die Entwicklung des Metaverse vorerst als Zukunftsmusik betrachten muss.

Mehrere Studien haben den Nutzen von VR und AR im Rahmen medizinscher An- wendungen untersucht. Dabei zeigt sich, dass vielerorts bereits außerhalb des urologischen Fachgebiets insbesondere im chirurgischen Sektor fest integrierte Lösungen bestehen, die bereits routinemäßig im Alltag etabliert sind. VR und AR können dabei auf unterschiedlichste Art und Weise positiven Einfluss auf die ärztliche Wahrnehmung ausüben, wobei mehrere Studien analysierten, dass insbesondere von den folgenden drei Anwendungsgebieten medizinischer Fortschritt erwartet wird $[3,6,7]$ :

1. intraoperative Einblendung bildgebender Verfahren

2. Telementoring und Telemedizin

3. anatomische und operative Ausbildung.

Dies verbunden mit dem Ziel, relevante Parameter wie Verringerung der Komplikationsraten, Kosteneinsparungen und präzisere chirurgische Ergebnisse mit entsprechend einhergehender verbesserter Lebensqualität und -erwartung für 


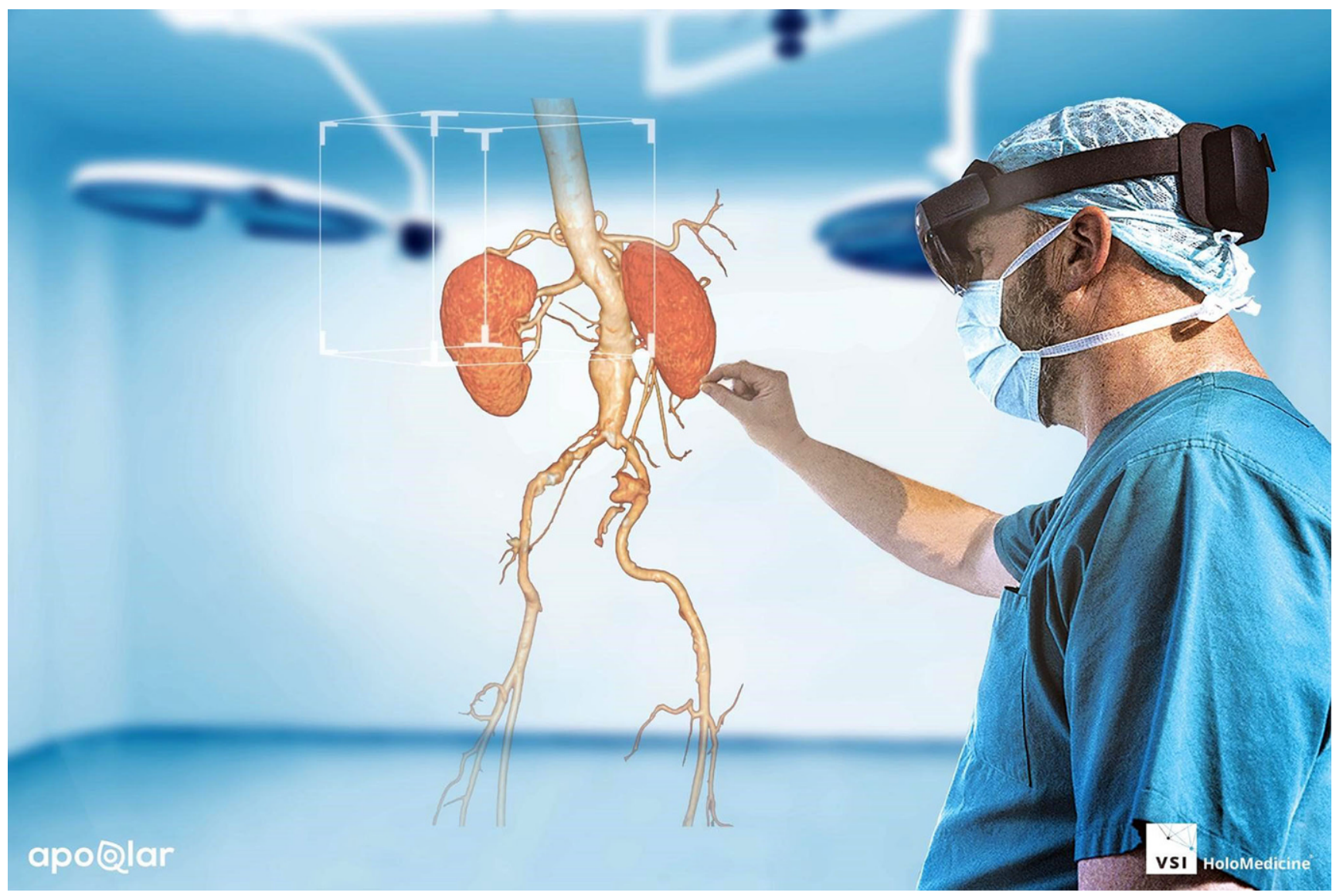

Abb. $2 \Delta$ Darstellung eines virtuellen Hologramms der Nieren mit Gefäßversorgung unter Verwendung der Mixed Reality Smartglass HoloLens II (Microsoft) und der Virtual Surgery Intelligence (VSI) Software von ApoQlar. (Mit freundl. Genehmigung, @apoQlar GmbH, alle Rechte vorbehalten)

die Patienten zu ermöglichen. Isoliert das Fachgebiet der Urologie betrachtet haben bereits viele KollegInnen in systematischen Reviews umfassend die bestehenden Anwendungsversuche beleuchtet und mussten hierbei festhalten $[1,6,8]$, dass zwar einige interessante Anwendungsideen und Ansätze in der Urologie bestehen, diese jedoch noch den Beweis schuldig bleiben, tatsächlich die urologische Versorgung hinsichtlich der oben genannten Parameter bzw. Endpunkte zu optimieren. Im Folgendem gehen wir auf die oben genannten drei vielversprechenden Anwendungsgebiete für die Implementierung von VR und AR im urologischen Alltag ein (• Tab. 1).

\section{Intraoperative Einblendung bildgebender Verfahren}

Viele Anwendungen im Bereich der VR und AR ermöglichen den Zugriff auf röntgenologische Bildgebung wie CT, MRT oder
Echtzeitsonographie während einer Operation unter aseptischen Bedingungen [9]. Die Idee hierbei beruht darin, dem Operationsteam intraoperativ ein Tool zur Verfügung zu stellen, welches ihnen patientenindividuelle Informationen über die Lokalisation von anatomischen oder pathologischen Strukturen liefert und somit das chirurgische Verständnis am "point of care“ erweitert [10]. Dies mit dem Ziel, präzisere operative Ergebnisse unter Schonung gesunder Strukturen zu ermöglichen. Um diesen intraoperativen kognitiven Transfer des Operateurs zwischen präoperativer Bildgebung und realen Strukturen weiter zu optimieren sind bereits sog. MatchingTools und Tracking-Tools entstanden. Diese ermöglichen ebenfalls am „point of care“ eine Fusion realer Organe/Körper mit patientenindividuellen virtuellen Hologrammen und sollen so die Navigation während der Operation erleichtern (- Abb. 2; [8]). Laut Yoon et al. [7] spielen genau diese Formen der AR-Anwendungen die Schlüs- selrolle in der Transformation zukünftiger Operationssäle.

Wake et al. [11] konfigurierten mittels Unity 3D-Software ein AR-Modell eines Nierentumors, welches über die Smartglass Hololens von Microsoft ${ }^{\circledR}$ (Redmond, Washington, USA) perioperativ visualisiert werden konnte und so Aufschluss über die Tumorlage, Infiltrationstiefe und Gefäßversorgung lieferte. Die Kollegen schlussfolgerten, dass die 3D-Visualisierung des Tumors die intraoperative Entscheidungsfindung bei Begutachtung des AR-Modells maßgeblich beeinflussen kann.

Porpiglia et al. [12] griffen diese Thematik ebenso in einer Reihe von Originalarbeiten auf. Für komplexe Nierentumoren mit einem PADUA-Score („preoperative aspects and dimensions used for an anatomical classification") $\geq 10$ wurden vor transperitonealer roboterassistierten Nierenteilresektion im Rahmen einer retrospektiven Studie mit insgesamt bei $48 \mathrm{~Pa}$ - 
Tab. 1 Tabellarische Übersicht über aktuelle urologische Forschungsprojekte im Bereich der Virtual Reality (VR) und Augmented Reality (AR) in den Anwendungsgebieten intraoperative Einblendung, Telementoring/Telemedizin und anatomische/operative Ausbildung

\begin{tabular}{|c|c|c|c|c|}
\hline Jahr & Autor & Operation & Technology/Verfahren & Resultat \\
\hline \multicolumn{5}{|c|}{ Intraoperative Einblendung bildgebender Verfahren } \\
\hline 2018 & $\begin{array}{l}\text { Wake et al. } \\
\text { [11] }\end{array}$ & $\begin{array}{l}\text { Robotische Nieren- } \\
\text { teilresektion }\end{array}$ & AR-Nierenmodelle via Unity 3D-Software & $\begin{array}{l}\text { Positiven Einfluss auf intraoperative } \\
\text { Entscheidungsfindung }\end{array}$ \\
\hline 2019 & Porpiglia et al. & $\begin{array}{l}\text { Robotische Nieren- } \\
\text { teilresektion }\end{array}$ & $\begin{array}{l}\text { AR-Modelle der Niere via „hyperaccuracy three-dimen- } \\
\text { sional reconstruction“ und mit intraoperativer Real-time- } \\
\text { Fusion/Matching }\end{array}$ & $\begin{array}{l}\text { AR-Nierenmodell ist konventionellen } \\
\text { Ultraschall überlegen }\end{array}$ \\
\hline 2018 & $\begin{array}{l}\text { Porpiglia et al. } \\
\text { [13] }\end{array}$ & $\begin{array}{l}\text { Robotische Pro- } \\
\text { statektomie }\end{array}$ & $\begin{array}{l}\text { AR-Prostatamodelle via „hyperaccuracy three-dimensional } \\
\text { reconstruction systems“ }\left(\mathrm{HA} 3 \mathrm{D}^{\mathrm{TM}}\right) \text { und intraoperatives } \\
\text { Matching via TilePro }{ }^{\mathrm{TM}} \text { multi-input display technology }\end{array}$ & $\begin{array}{l}\text { Suffizientes Matching, das individua- } \\
\text { lisierte operative Therapie ermög- } \\
\text { licht }\end{array}$ \\
\hline 2021 & Porpiglia et al. & $\begin{array}{l}\text { Robotische Pro- } \\
\text { statektomie }\end{array}$ & $\begin{array}{l}\text { AR-Prostatamodelle mit intraoperativem autonomem } \\
\text { Matching via Artificial Intelligence Convolutional Neuronal } \\
\text { Network Software }\end{array}$ & $\begin{array}{l}\text { Intraoperative Fusion verbessert } \\
\text { onkologisches Ergebnis bei fortge- } \\
\text { schrittenen Tumoren }\end{array}$ \\
\hline 2018 & $\begin{array}{l}\text { Schiavina } \\
\text { et al. [15] }\end{array}$ & $\begin{array}{l}\text { Robotische Pro- } \\
\text { statektomie }\end{array}$ & Intraoperatives Matching von 3D-AR-Prostatamodellen & $\begin{array}{l}\text { Ermöglicht Optimierung und Anpas- } \\
\text { sung beim "nerve-sparing" }\end{array}$ \\
\hline 2020 & $\begin{array}{l}\text { Janabi et al. } \\
\text { [16] }\end{array}$ & Ureterorenoskopie & $\begin{array}{l}\text { Smartglass AR-assistierter (HoloLens)Ersatz der koventio- } \\
\text { nellen Monitore (Endoskopie/Fluoroskopie) }\end{array}$ & $\begin{array}{l}\text { Smartglass AR-Ureterorenoskopie } \\
\text { ist durchführbare alternative; ggf. } \\
\text { bessere Ergonomie }\end{array}$ \\
\hline 2018 & Qian et al. [17] & $\begin{array}{l}\text { Robotische Opera- } \\
\text { tionen }\end{array}$ & $\begin{array}{l}\text { Smartglass AR-assistierte (HoloLens)Ersatz des koven- } \\
\text { tionellen Monitors des Bedside-Assistenten während } \\
\text { daVinci-Operation }\end{array}$ & $\begin{array}{l}\text { 3D-Sichtverhältnisse und bessere } \\
\text { Augen-Hand-Koordination für Assis- } \\
\text { tenten }\end{array}$ \\
\hline \multicolumn{5}{|c|}{ Telementoring und Telemedizin } \\
\hline 2021 & $\begin{array}{l}\text { El-Asmar et al. } \\
\text { [22] }\end{array}$ & $\begin{array}{l}\text { Aquablation der } \\
\text { Prostata }\end{array}$ & $\begin{array}{l}\text { Supervision Aquablation der Prostata via Proximie-Platt- } \\
\text { form und AR-Telementoring durch Remote Surgeon }\end{array}$ & $\begin{array}{l}\text { Telementoring sichere und kostenef- } \\
\text { fektive Unterstützung }\end{array}$ \\
\hline \multicolumn{5}{|c|}{ Anatomische und operative Ausbildung } \\
\hline 2021 & $\begin{array}{l}\text { Schwartzmann } \\
\text { et al. }\end{array}$ & Anatomieunterricht & $\begin{array}{l}\text { Visible Body = Tablet- oder Smartphone-basierte AR- } \\
\text { Applikation mit Generierung virtueller Organmodelle }\end{array}$ & $\begin{array}{l}\text { Erhöhtes Engagement und bessere } \\
\text { räumliche Vorstellung }\end{array}$ \\
\hline 2020 & $\begin{array}{l}\text { Schoeb et al. } \\
\text { [4] }\end{array}$ & $\begin{array}{l}\text { Studentenpraktikum } \\
\text { Blasenkatheterismus }\end{array}$ & $\begin{array}{l}\text { Smartglass MR-assistierte (HoloLens)Applikation mit } \\
\text { praktischer Anleitung eines Blasenkatheters }\end{array}$ & $\begin{array}{l}\text { Effiziente und kostengünstige Über- } \\
\text { mittlung von Lerninhalten }\end{array}$ \\
\hline 2019 & $\begin{array}{l}\text { Yamada et al. } \\
\text { [19] }\end{array}$ & Nierentumor & $\begin{array}{l}\text { 3D-Rekonstruktion der Nieren mit Holoeyes-Software und } \\
\text { AR-Visualisierung via Smartglass Mirage Solo }\end{array}$ & $\begin{array}{l}\text { Unkomplizierte AR-3D-Rekonstrukti- } \\
\text { on zur präoperative Planung }\end{array}$ \\
\hline 2019 & $\begin{array}{l}\text { Yoshida et al. } \\
\text { [19] }\end{array}$ & Nierentumor & $\begin{array}{l}\text { 3D-Rekonstruktion der Nieren mit Holoeye-Software und } \\
\text { AR-Visualisierung via Smartglass HoloLens }\end{array}$ & $\begin{array}{l}\text { AR-3D-Rekonstruktion beeinflusst } \\
\text { die präoperative Planung }\end{array}$ \\
\hline 2017 & Porpiglia et al. & Nierentumor & $\begin{array}{l}\text { AR-Modelle via „hyperaccuracy three-dimensional recon- } \\
\text { struction“ bei komplexen Nierentumoren }\end{array}$ & $\begin{array}{l}\text { Präoperative AR-Modelle senken die } \\
\text { intraoperative Notwendigkeit für } \\
\text { eine globale Ischämie }\end{array}$ \\
\hline 2018 & $\begin{array}{l}\text { Neumann } \\
\text { et al. [31] }\end{array}$ & TURB & VR-TURB via UroTrainer bei Studenten & $\begin{array}{l}\text { Effektivität und Sicherheit einer VR- } \\
\text { TURB werden durch Training rasch } \\
\text { gesteigert }\end{array}$ \\
\hline 2018 & Schulz et al. & TURB & $\begin{array}{l}\text { VR-TURB via UroTrainer bei urologischen Assistenz- und } \\
\text { Fachärzten }\end{array}$ & $\begin{array}{l}\text { Komplettiert die endurologische } \\
\text { Ausbildung }\end{array}$ \\
\hline
\end{tabular}

tienten ein AR-Modell der tumortragenden Niere mittels "hyperaccuracy threedimensional reconstruction" konfiguriert, welches dann intraoperativ während Tumorresektion virtuell mit der realen Niere fusioniert wurde. Als Vergleichsgruppe diente ein Arm mit 43 Patienten, bei denen nur ein konventioneller intraoperativer Ultraschall angewendet wurde. Unter dieser 3D-AR-Führung wurden weniger globale Ischämien, bessere Enukleationsraten und weniger Verletzungen des Hohlsystems beobachtet. Zudem wurde interessanterweise 3 Monate postoperativ im Rahmen des CT-Kontrollstagings ebenso eine besser erhaltene Nierenperfusion festgehalten.

\section{》) 3D-AR-Nierenmodelle für komplexe Tumore könnten dem konventionellen Ultraschall überlegen sein}

Die Kollegen reüssierten, dass diese roboterassistierte Nierenteilresektion unter Fusion (alt. Matching) mit individuellen 3D-
AR-Nierenmodellen für komplexe Tumore dem konventionellen Ultraschall überlegen ist. Zudem führte ihre AR-Fusion zu einer präziseren Resektion, weniger postoperativen Komplikationen und besseren funktionellen Ergebnissen.

Einen ähnlichen Ansatz übertrugen dieselbe Arbeitsgruppe um Porpiglia 2019 [13] auf die roboterassistierte radikale Prostatektomie und führten diese ebenso nach Erstellung eines AR-Modells der Prostata unter Verwendung des oben genannten "hyperaccuracy three-dimen- 
sional reconstruction system" (HA3D ${ }^{\mathrm{TM}}$, MEDICS, Turin, Italien) durch. Hierbei wurde die hochauflösende multiparametrische MRT (mpMRT; 1-mm-Schichten) der Prostata bei insgesamt 30 Patienten nach AR-Konfiguration in die da VinciKonsole (Intuitive Surgical, Sunnyvale, USA) eingespeist und virtuell durch den Operateur mittels TilePro $^{\text {TM }}$ Multi-Input Display Technology (Intuitive Surgical) mit der realen Prostata fusioniert und bei insgesamt vier Schlüsselstellen zur intraoperativen Navigation eingeblendet. Zusätzlich wurde intraoperativ bei allen 30 Patienten die Indexläsion nach ARFusion durch den Operateur mittels eines Clips markiert. Die finale histopathologische Beurteilung bestätigte bei allen Patienten die korrekte intraoperative Markierung auf der Höhe der tatsächlichen Indexläsion. Auch hier suggerierten die Kollegen, dass eine intraoperative Echtzeitfusion mit 3D-AR-Prostatamodellen dem operativen Ergebnis insbesondere durch die Möglichkeit der individualisierten Anpassungen Vorteile bringen könnte [13]. Basierend auf diesen Forschungs- ergebnissen entwickelten die Kollegen Anfang 2021 ein Verfahren, welches unter Verwendung einer Artificial Intelligence Convolutional Neuronal Network Software automatisch eine intraoperative Fusion über die da Vinci-Konsole konfiguriert. Bei 10 Patienten mit Verdacht auf ein extrakapsuläres Wachstum konnte hier in 8 von 10 Fällen ein Stadium pT3a/b bestätigt werden, und die zusätzlich anhand des AR-Modells der Prostata intraoperativ entnommenen Biopsien zeigten in 75\% einen Tumornachweis. Entsprechend wurde auch hier bei lokal fortgeschrittenen Tumoren angenommen, dass das onkologische Ergebnis durch eine intraoperative AR-Fusion verbessert werden kann [14].

Auch im Rahmen des "nerve-sparing“ scheint diese Form der AR-unterstützten roboterassistierten Prostatektomie zukünftig Vorteile bieten zu können. Schiavina et al. [15] konnten hier an 26 Patienten, die sich einem nerverhaltenden Verfahren einer Prostatektomie unterzogen, demonstrieren, dass die intraoperative Einblendung des AR-Prostatamodells basierend auf dem präoperativen
mpMRT die intraoperative Identifikation der Indexläsion unterstützt und in 1 von 3 Patienten sogar zu einer Anpassung des chirurgischen Vorgehens beim "nervesparing" führt.

Die AR bietet zudem die Option, den Monitor im Rahmen vieler endoskopischen Operationen zu ersetzen. Janabi et al. [16] haben dies im Rahmen der Ureterorenoskopie bei insgesamt 72 Teilnehmern (Novizen bis Experten) erforscht. Hierbei erfolgte die videoskopische Bildeinblendung über den Display der Smartglass Hololens, wobei zusätzlich über die Smartglass das präoperative CT und auch die intermittierend durchgeführte konventionelle röntgenologische Fluoroskopie eingeblendet werden konnte.

Neben der Idee den Operateur im Rahmen roboterassistierter Operationen mittels $A R$ und VR zu unterstützen gibt es bereits Projekte, bei welchen die Assistenten (ARssist) mit einer Smartglass ausgestattet wird. Die Smartglass Hololens von Microsoft ${ }^{\circledR}$ ermöglicht dabei im Versuchsaufbau von Qian et al. [17] dem Assistenten neben der 3D-Sicht entspre-

Hier steht eine Anzeige. 
chend der da Vinci-Konsole v. a. eine freie Auge-Hand-Koordination, die es ihm beispielsweise erlaubt, gleichzeitig die Clipzange einzuführen und parallel diese direkt intrakorporal weiter zu verfolgen, ohne dabei ungelenk auf einen Monitor blicken zu müssen. Zudem haben Qian et al. ein AR-unterstütztes Modul programmiert, das dem Assistenten über die Smartglass virtuell den geometrischen Verlauf der Roboterarme auf/in den Körper projiziert, um inm die räumliche Lagebeziehung zwischen Operationsgebiet und Endoskopen besser zu verdeutlichen.

Aktuell konzentrieren sich die meisten Applikationen der intraoperativen Bildeinblendung auf laparoskopische Verfahren. Jedoch ist davon auszugehen, dass auch hier der Transfer auf die klassische offene Chirurgie erfolgen wird. Das deutsche Unternehmen ApoQlar hat sich dabei auf eben dieses virtuelle Matching radiologischer Bildgebung mit realen Körpern/ Organen spezialisiert und bereits v.a. in chirurgischen Fachgebieten seinen Nutzen demonstrieren können (• Abb. 2).

\section{Telementoring und Telemedizin}

Telementoring und Telemedizin sind seit Jahren viel diskutierte Konzepte, die basierend auf innovativen Weiterentwicklungen und dem zunehmend technikaffinem Patientengut an Interesse zunehmen. Während die Telemedizin mehr die Interaktion zwischen Arzt und Patient, welche sich an geographisch unterschiedlichen Orten befinden, durch ein technologisches Kommunikationsmittel beschreibt, so ist gleichermaßen das Telementoring als Anleitung eines Auszubildenden über die Ferne hinweg zu verstehen [18].

\section{I) Viele ambulante urologische Patienten begrüßen routinemäßige ärztliche Konsultation via Telemedizin}

Im Rahmen der COVID-19-Pandemie ("COronavirus disease $2019^{\prime \prime}$ ) konnte in einer Studie von Böhm et al. [19] demonstriert werden, dass $84,7 \%$ der ambulanten urologischen Patienten einer deutschen Klinik inre anstehende routinemäßige ärztliche Konsultation via Telemedizin bevorzugen würden. Dabei zeigen die bestehenden telemedizinische Produkte eine hohe Patientenzufriedenheit bei einfacher Handhabung [20].

Glenn et al. [21] demonstrierten zudem bereits 2017 in einer Umfrage unter Mitgliedern des American College of Surgeons, dass ca. $80 \%$ der Chirurgen im ländlichen Raum unabhängig vom Ausbildungsgrad Interesse an Telementoring-/ Monitoring-Programmen bekunden. Dies insbesondere mit der Intention neue Operationstechniken zu erlernen und im Falle unerwarteter intraoperativer Befunde einen externen Experten konsultieren zu können. Diese Versorgungslücken könnten mit Hilfe von VR- oder AR-Anwendungen zukünftig unter sehr authentischen Bedingungen geschlossen werden.

Die Firma Proximie ist einer der Vorreiter in der Welt des Telementorings mit $>5000$ chirurgischen Eingriffen. Proximie ist dabei eine innovative digitale Plattform, die AREchtzeitvideostreams verwendet, um bis zu 40 Teilnehmer gleichzeitig an einer Operation virtuell teilnehmen zu lassen [22]. Die Plattform besteht aus einem mobilen Modul mit mehreren Computer-, Kameraund Audiosystemen, die in jeden Operationssaal integriert werden kann, wobei das Modul zusätzlich simultan an die intraoperativ verwendeten Endoskope oder Videokameras konnektiert wird. Der entfernt sitzende Chirurg („Remote Surgeon“) leitet den auszubildenden Kollegen an, indem er direkt verbal Feedback gibt und zusätzlich manuell über das Display visualisierbar für den Kollegen via AR eingreift, beispielsweise um bestimmte Landmarken zur Orientierungshilfe zu setzten. Der Remote Surgeon hat dabei volle Navigationskontrolle über das Proximie-Modul und kann den kompletten Operationssaal aus der Vogelperspektive überblicken [22].

El-Asmar et al. [22] demonstrierten unter Verwendung der Proximie-Plattform die primäre Anlernung einer Aquablation der Prostata. Eine Aquablation wurde hier bei insgesamt 38 Patienten klassisch am Patienten und bei 21 Patienten mittels dreier Remote Surgeons via Telementoring angeleitet. Die primären Endpunkte Operationszeit, postoperative Katheterentfernung, Hämoglobinabfall, Ischurie und unerwünschte Nebenwirkungen waren dabei in den beiden Vergleichsgruppen statistisch nicht signifikant unterschiedlich.
Die Autoren schlussfolgerten, dass gerade nach der klassischen Supervision am Patienten die Einführungsphase neuer chirurgischer Verfahren durch das Telementoring sicher und kosteneffektiv unterstützt werden kann.

\section{Anatomische und operative Ausbildung}

Die VR- und AR-Applikationen werden zukünftig den Umgang mit Lerninhalten revolutionieren. Anatomische Strukturen und pathophysiologische Prozesse können bereits mittels VR und AR lehrreich illustriert werden und ermöglichen zudem oftmals den interaktiven teils spielerischen Austausch mit den jeweiligen Lektionen. Moro et al. [23] beobachteten dabei bei Studenten, denen zusätzlich zum klassischen Anatomieunterricht derlei VR- und AR-Applikationen zur Verfügung standen, neben einer engagierteren Lernbereitschaft eine erhöhte Lerneffizienz. Zudem haben mehrere Forschungsgruppen aufzeigen können, dass der Einsatz von VRund AR-Trainingseinheiten im Rahmen der operativen Ausbildung von Novizen zu einer Reduktion der Operationszeit und Komplikationsrate, sowie zu einer verbesserten chirurgischen Präzision führen [1, 6]. Zwar werden VR- und AR-Anwendungen den herkömmlichen anatomischen und operativen Ausbildungsprozess niemals ersetzen, jedoch kann dieser rascher und sicherer erfolgen.

\section{》) Die VR- und AR-Applikationen werden zukünftig den Umgang mit Lerninhalten revolutionieren}

Einer der wesentlichen Bestandteile der vorklinischen Ausbildung stellt der klassische Anatomieunterricht dar. Obwohl das Fach selbst sehr lernintensiv ist, so ist die Zeit im Anatomiesaal meist zu kurz. Viele innovative AR- und VR-Lernplattformen stehen als E-Resource bereits meist kommerziell zur Verfügung und sollten nach den positiven Resultaten der Metaanalyse von Bölek et al. [24] den klassischen Anatomieunterricht im Zuge der fortschreitenden Technisierung ergänzen, wovon auch die urologische studentische Ausbildung mit seiner teils komplexen Anatomie im kleinen Becken profitieren würde. Nach 
McClurg et al. [25] ist hier u.a. die E-Resource-Plattform Visible Body ${ }^{\circledR}$ im Fokus. Visible Body ${ }^{\circledR}$ ist dabei eine AR-Applikation, die es dem Anwender erlaubt über ein Tablet oder Smartphone 3D-Simulationen der kompletten menschlichen Anatomie inklusive der Mikroanatomie direkt in das Lernumfeld zu projizieren [26]. Die virtuellen Organmodelle können dann manuell skaliert und bearbeitet werden mit teils zusätzlich vermerkten Querverweisen und ermöglichen so ein optimales räumliches anatomisches Verständnis.

Die praktische Ausbildung von Studenten kosteneffektiv zu gestalten kann zudem über ein MR-Tool mit Smartglass gelingen. Schoeb et al. [4] haben dafür eine MR-Applikation konfiguriert die „stepby-step" mittels auditiven und visuellen Anweisungen Studenten ( $n=57)$ über die Smartglass Hololens den Blasenkatheterismus lehrt und praktisch anleitet. In ihrer randomisierten Studie stellten sie der MR-Gruppe 107 Studenten gegenüber, die klassisch durch einen Ausbilder im Rahmen eines Seminars angeleitet wurden. Beide Gruppen wurden standardisiert nach OSCE-Prüfung („objective structured clinical examination") bewertet. Dabei schlossen die Studenten der MR-Gruppe statistisch signifikant besser $a b$, weshalb auch diese vermeintlich simplen Anwendungen ein großes Potenzial für derlei Applikationen zu sein scheinen mit dem Ziel, kosteneffektiv, interessant und effektiv Lehrinhalte zu vermitteln.

Im Rahmen der präoperativen Vorbereitung auf eine partielle Nierenteilresektion ist die Kenntnis über Tumorlokalisation, Infiltrationstiefe, Gefäßversorgung und Lagebeziehung zum Hohlsystem essenziell. Unlängst ist hier bekannt, dass eine 3D-Rekonstruktion beispielsweise durch ein Hologramm wichtige Information für die präoperative Planung liefern kann und der alleinigen Betrachtung eines planaren CT oder MRT überlegen ist [27]. Nach Yamada et al. [28] kann eine 3DRekonstruktion eines CT mit Holoeyes Software (Tokyo, Japan) in ein virtuelles Hologramm der Niere mit allen oben genannten Informationen unkompliziert erfolgen. Dies stand dem Operateur präoperativ über die VR-Smartglass Mirage Solo (Lenovo Hong Kong, China) zur operativen Planung und Visualisierung bereit.
Diese 3D-Rekonstruktion der Holoeyes XR Software untersuchten auch Yoshida et al. [29] bei 8 Urologen mit Projektion von Nierentumormodellen in die Smartglass Hololens und suggerierten das die präoperative Entscheidungsfindung über den operativen Ablauf maßgeblich beeinflusst werden kann.

Auch dieses Thema griffen Porpiglia et al. auf. Zwischen 2016-2017 entwickelten sie für roboterassistierte Nierenteilresektionen bei Nierentumoren mit einem PADUA-Score $\geq 10$ ein präoperatives ARbasiertes Simulationsmodell, welches eine selektive Ischämie intraoperativ ermöglichen sollte und stellten dieses Verfahren einer konventionellen Vergleichsgruppe gegenüber. Dabei konnten sie für $21 \mathrm{~Pa}$ tienten, bei denen mittels "hyperaccuracy three-dimensional reconstruction" ein AR-Modell angefertigt worden war zeigen, dass die Notwendigkeit intraoperativ eine globale Ischämie durchzuführen wesentlich geringer war. In der Vergleichsgruppe von 31 Patienten ohne präoperatives ARModell waren es $80 \%$ gegenüber nur $24 \%$ mit AR-Rekonstruktion [30].

Die transurethrale Resektion der Harnblase (TURB) stellt einen der ersten endoskopischen Eingriffe im urologischen Weiterbildungscurriculum dar. Neumann et al. [31] stellten dabei in ihrem VR-Trainingsprogramm (UroTrainer, Karl Storz $\mathrm{GmbH}$, Walsdorf) bei Medizinstudenten fest, dass bereits wenig Übung die Effektivität und Sicherheit einer VR-TURB stark erhöhen kann. Ein Transfer von VR-TURB in eine reale TURB hat in dem genannten Versuchsaufbau allerdings nicht stattgefunden. Schulz et al. [32] jedoch konnten unter Anwendung des gleichen VR-Trainingssimulators UroTrainer bei 22 urologischen Assistenz- und Fachärzten zeigen, dass der VR-Trainer subjektiv und objektiv das für TURB optimiert und deswegen zur Vervollständigung der endurologischen Ausbildung angewendet werden sollte.

\section{Ausblick}

Die VR- und AR-Anwendungen werden zunehmend in den medizinischen Alltag integriert werden. Die vielversprechendsten Anwendungsgebiete in der Urologie betreffen dabei die intraoperative Bildeinblendung, Telemedizin/Telementoring und die anatomische und operative Ausbildung. Hierfür haben bereits heute viele innovative urologische Forschungsgruppen technische Lösungen konzeptioniert.

Im Rahmen der intraoperativen Bildeinblendung bestehen bereits insbesondere bei der Nierenteilresektion bzw. Prostatektomie interessante VR- und AR-Möglichkeiten, die Telemedizin/Telementoring wird zunehmend durch innovative Lösungen vereinfacht für jedermann zugänglicher und die ärztliche Ausbildung wird v.a. durch realitätsgetreue 3D-Szenarien eine neue Dimension erfahren.

Abschließend muss angemerkt werden, dass zum gegenwärtigen Zeitpunkt wenige Studien vorliegen, die einen definitiven Nutzen für eine bestimmte AR- oder VR-Anwendung im urologischen Fachgebiet ausweisen. Allerdings verdichten sich die zunehmend positiven wissenschaftlichen Ergebnisse, sodass die Integration von VR- und AR-Anwendungen in die klinische Routine nur eine Frage der Zeit ist. Die Urologie war jeher ein innovatives Fachgebiet und wird auch diesen Wandel weiter mitgestalten.

\section{Fazit für die Praxis}

- VR (Virtual Reality) und AR (Augmented Reality) werden zunehmend in den medizinischen Alltag integriert.

- Die vielversprechendsten Anwendungsgebiete in der Urologie betreffen dabei die intraoperative Bildeinblendung, Telemedizin/Telementoring und die anatomische/operative Ausbildung.

- VR (Virtual Reality) ist eine vollständig künstliche von Computern simulierte und generierte Umgebung. Die AR (Augmented Reality) hingegen generiert keine rein virtuelle Umgebung, sondern ergänzt die reale Welt mit virtuellen Informationen, während die Mixed Reality (MR) Attribute dieser beiden Welten vereint.

- Smartglasses sind Datenbrillen die visuelle und auditive Sensationen dem Anwender bereitstellen. Hierüber können virtuelle Bilder oder Modelle in das Sichtfeld des Anwenders unter aseptischen Bedingungen projiziert werden.

- Dreidimensionale VR und AR Organmodelle können das anatomische Verständnis verbessern und die intraoperative Entscheidungsfindung beeinflussen.

- VR- und AR-Applikationen werden zukünftig den Umgang mit Lerninhalten revolutionieren. 


\section{Korrespondenzadresse}

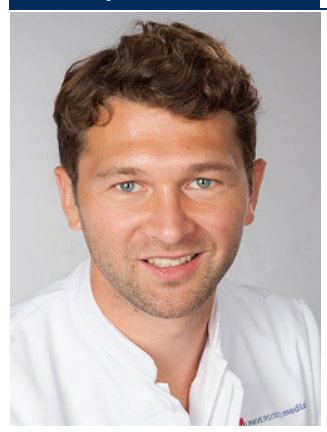

\section{Dr. P. Sparwasser}

Department of Urology, University Medical Center, Johannes Gutenberg University Langenbeckstr. 1, 55131 Mainz, Deutschland Peter.sparwasser@unimedizin-mainz.de

\section{Einhaltung ethischer Richtlinien}

Interessenkonflikt. P. Sparwasser, M. Haack, L. Frey, A. Haferkamp und H. Borgmann geben an, dass kein Interessenkonflikt besteht.

Für diesen Beitrag wurden von den Autoren keine Studien an Menschen oder Tieren durchgeführt. Für die aufgeführten Studien gelten die jeweils dort angegebenen ethischen Richtlinien.

\section{Literatur}

1. Bertolo R, Hung A, Porpiglia F et al (2020) Systematic review of augmented reality in urological interventions: the evidences of an impact on surgical outcomes are yet to come. World J Urol 38:2167-2176. https://doi.org/10. 1007/s00345-019-02711-z

2. Khor WS, Baker B, Amin K et al (2016) Augmented and virtual reality in surgery - the digital surgical environment: applications, limitations and legal pitfalls. Ann Transl Med 4:23454

3. Borgmann H, Rodriguez Socarras M, Salem J et al (2017) Feasibility and safety of augmented realityassisted urological surgery using smart-glass. World JUrol 35:967-972

4. Schoeb DS, Schwarz J, Hein S, Schlager D, Pohlmann PF, Frankenschmidt A, Gratzke C, MiernikA (2020)Mixed reality for teaching catheter placement to medical students: a randomized single-blinded, prospective trial. BMC Med Educ 20(1):510. https://doi.org/10.1186/s12909-02002450-5

5. Hurtz S (2021) Facebook will 10000 neue Jobs in Europa schaffen. https://www.sueddeutsche. de/wirtschaft/facebook-metaverse-europa- 1 . 5442647.Zugegriffen: 15. Nov. 2021

6. Sparwasser PM, Schoeb D, Miernik A, Borgmann H (2018) Augmented Reality und Virtual Reality im Operationssaal - Status Quo und Quo vadis. Aktuelle Urol 49(6):500-508. https://doi.org/10. 1055/a-0759-0029

7. Yoon JW, Chen RE, Kim EJ et al (2018) Augmented reality for the surgeon: systematic review. Int J Med Robot 14:e1914

8. Reis G, Yilmaz M, Rambach J, Pagani A, SuarezIbarrola R, Miernik A, Lesur P, Minaskan N (2021) Mixed reality applications in urology:

\section{Virtual and augmented reality in urology}

Although continuous technological developments have optimized and evolved medical care throughout time, these technologies were mostly still comprehensible for users. Driven by immense financial efforts, modern innovative products and technical solutions are transforming medicine today and will do so even more in the future: virtual and augmented reality. This review critically summarizes the current literature and future uses of virtual and augmented reality in the field of urology.

\section{Keywords}

Mixed reality $\cdot$ Smart glasses $\cdot$ Augmented reality $\cdot$ Telemedicine $\cdot$ Matching requirements and future potential. Ann Med Surg (Lond) 13(66):102394. https://doi.org/10.1016/j. amsu.2021.102394

9. van Oosterom MN, van der Poel HG, Navab N, van de Velde CJH, van Leeuwen FWB (2018) Computerassisted surgery: virtual- and augmented-reality displays for navigation during urological interventions. Curr Opin Urol 28(2):205-213. https://doi. org/10.1097/MOU.0000000000000478

10. SchoebDS, Rassweiler J, Sigle A etal (2021) Robotik und intraoperative Navigation. Urologe 60:27-38. https://doi.org/10.1007/s00120-020-01405-4

11. Wake N, Bjurlin MA, Rostami P et al (2018) Threedimensional printing and augmented reality: enhanced precision for robotic assisted partial nephrectomy. Urology 116:227-228

12. Porpiglia $F$, Checcucci $E$, Amparore $D$, Piramide $F$ Volpi G, Granato S, Verri P, Manfredi M, Bellin A, Piazzolla P, Autorino R, Morra I, Fiori C, Mottrie A (2020) Three-dimensional augmented reality robot-assisted partial nephrectomy in case of complex tumours (PADUA $\geq 10$ ): a new intraoperative tool overcoming the ultrasound guidance. Eur Urol 78(2):229-238. https://doi.org/ 10.1016/j.eururo.2019.11.024

13. Porpiglia F, Checcucci E, Amparore D et al (2018) Augmented-reality robot-assisted radical prostatectomy using hyper-accuracy threedimensional reconstruction $\left(H A 3 D^{T M}\right)$ technology: a radiological and pathological study. BJU Int. https://doi.org/10.1111/bju.14549

14. Porpiglia F, Checcucci E, Amparore D, De Cillis S, Volpi G, Massa F, Bollito E, Piazzolla P, Bellin A, Manfredi M, VezzettiE, FioriC (2021)A new tool able to reduce the positive surgical margin rate during robotic prostatectomy: artificial intelligence 3D augmented reality guided selective biopsy

15. Schiavina R, Bianchi L, Lodi S, Cercenelli L, Chessa F, BortolaniB, GaudianoC,CasablancaC,DroghettiM, Porreca A, Romagnoli D, Golfieri R, Giunchi F, Fiorentino $M$, Marcelli $E$, Diciotti $S$, Brunocilla $E$ (2020) Real-time augmented reality threedimensional guided robotic radical prostatectomy: preliminary experience and evaluation of the impact on surgical planning. Eur Urol Focus. https://doi.org/10.1016/j.euf.2020.08.004

16. Al Janabi HF, Aydin A, Palaneer S, Macchione N, AlJabir A, Khan MS, Dasgupta P, Ahmed K (2020) Effectiveness of the HoloLens mixed-reality headset in minimally invasive surgery: a simulation-based feasibility study. Surg Endosc 34(3):1143-1149. https://doi.org/10.1007/s00464-019-06862-3

17. Qian L, Deguet A, Kazanzides P (2018) ARssist: augmented reality on a head-mounted display for the first assistant in robotic surgery. Healthc Technol Lett 5(5):194-200. https://doi.org/10. 1049/htl.2018.5065
18. Erridge $S$, Yeung DKT, Patel HRH, Purkayastha $S$ (2019) Telementoring of surgeons: a systematic review. Surg Innov 26(1):95-111. https://doi.org/ $10.1177 / 1553350618813250$

19. Boehm K, Ziewers $S$, Brandt MP, Sparwasser $P$, Haack M, Willems F, Thomas A, Dotzauer R, Höfner T, Tsaur I, Haferkamp A, Borgmann H (2020) Telemedicine online visits in urology during the COVID-19 pandemic-potential, risk factors, and patients' perspective.EurUrol 78(1):16-20.https:// doi.org/10.1016/j.eururo.2020.04.055

20. Struck JP, Wenzel M, Heidenreich A, Merseburger A, Salem J (2018) Die Implementierung der Telemedizin im urologischen Alltag. Aktuelle Urol 49(6):509-514. https://doi.org/10.1055/a-07416664

21. Glenn IC, Bruns NE, Hayek D et al (2017) Rural surgeons would embrace surgical telementoring for help with difficult cases and acquisition of new skills. Surg Endosc 31:1264-1268

22. El-Asmar JM, Labban M, El-Hajj A(2021) Integration of aquablation through telemetry: an alternative to onsite proctoring? World J Urol 39:3473-3479. https://doi.org/10.1007/s00345-021-03603-x

23. Moro C, Stromberga Z, Raikos A et al (2017) The effectiveness of virtual and augmented reality in health sciences and medical anatomy. Anat Sci Educ 10:549-559

24. Bölek KA, De Jong G, Henssen D (2021) The effectiveness of the use of augmented reality in anatomy education: a systematic review and metaanalysis. Sci Rep 11(1):15292. https://doi.org/10. 1038/s41598-021-94721-4

25. McClurg C, Stieda V, Talsma N (2015) Comparing subscription-based anatomy E-resources for collections development. Med Ref Serv Q 34(3):282-295. https://doi.org/10.1080/ 02763869.2015 .1052690

26. Schwartzman G, Ramamurti P (2021) Visible body human anatomy atlas: innovative anatomy learning. JDigit Imaging 34(5):1328-1330. https:// doi.org/10.1007/s10278-021-00496-5

27. Antonelli A, Veccia A, Palumbo C et al (2018) Holographic reconstructions for preoperative planning before partial nephrectomy: a head-tohead comparison with standard CT scan. Urol Int 12:1-6. https://doi.org/10.1159/000495618

28. Yamada $Y$, Inoue $Y$, Kaneko $M$, Fujihara $A$, Hongo F, Ukimura O (2019) Virtual reality of threedimensional surgical field for surgical planning and intraoperative management. Int J Urol 26(9):942-943. https://doi.org/10.1111/iju.14047

29. Yoshida S, Sugimoto M, Fukuda S, Taniguchi N, Saito K, Fujii Y (2019) Mixed reality computed tomography-based surgical planning for partial nephrectomy using a head-mounted holographic 
computer. Int J Urol 26(6):681-682. https://doi. org/10.1111/iju.13954

30. Porpiglia F, Fiori C, Checcucci Eetal (2018) Hyperaccuracy three-dimensional reconstruction is able to maximize the efficacy of selective clamping during robot-assisted partial nephrectomy for complex renal masses. Eur Urol. https://doi.org/10.1016/j. eururo.2017.12.027

31. Neumann E, Mayer J, Russo Gl, Amend B, Rausch S, Deininger S, Harland N, da Costa IA, Hennenlotter J, Stenzl A, Kruck S, Bedke J (2019) Transurethral resection of bladder tumors: next-generation virtual reality training for surgeons. Eur Urol Focus 5(5):906-911. https://doi.org/10.1016/j.euf.2018. 04.011

32. Schulz GB, Grimm T, Buchner A, Jokisch F, Casuscelli J, Kretschmer A, Mumm JN, Ziegelmüller B, Stief CG, Karl A (2019) Validation of a high-end virtual reality simulator for training transurethral resection of bladder tumors. J Surg Educ 76(2):568-577. https://doi.org/10.1016/j.jsurg. 2018.08.001

\section{DEUTSCHER KREBSKONGRESS}

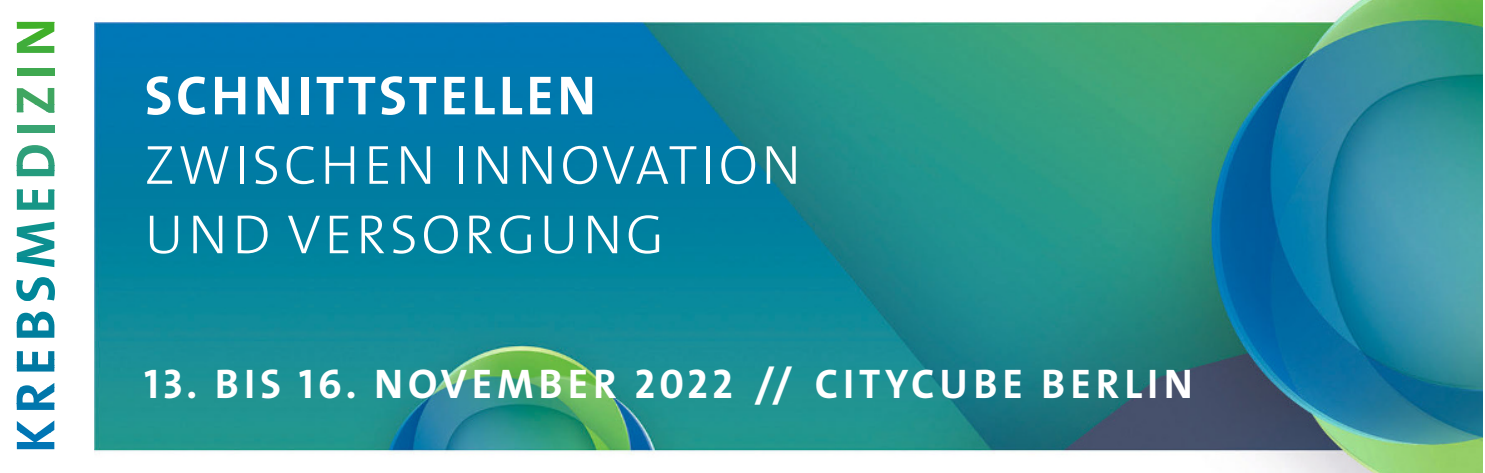

